Bull. Soc. math. France

131 (2), 2003, p. $211-228$

\title{
ÉQUATION DES ONDES AMORTIES \\ DANS UN DOMAINE EXTÉRIEUR
}

\author{
PAR Moez KHENISSI
}

\begin{abstract}
RÉsumé. - On étudie la position des pôles de diffusion du problème de Dirichlet pour l'équation des ondes amorties du type $\partial_{t}^{2}-\Delta+a(x) \partial_{t}$ dans un domaine extérieur. Sous la condition $\mathrm{du}$ " contrôle géométrique extérieur », on déduit alors le comportement des solutions en grand temps. On calcule en particulier le meilleur taux de décroissance de l'énergie locale en dimension impaire d'espace.

Abstract (Dissipative wave equation in an exterior domain). - We study the position of diffusion poles for the Dirichlet problem for the dissipative wave equation in the exterior of an arbitrary obstacle in $\mathbb{R}^{d}$. We deduce under the "Exterior Geometric Control" condition the behavior of the solutions for large time. We give, in particular, a formula for the best rate of decay of the local energy in odd dimension spaces.
\end{abstract}

\section{Introduction}

On s'intéresse dans cet article à l'équation des ondes amorties dans un domaine extérieur avec conditions de Dirichlet. On considère un obstacle borné $\Theta$ de $\mathbb{R}^{d}$ à bord $C^{\infty}$, on note $\Omega=\bar{\Theta}^{c}$ et $a(x) \in C_{0}^{\infty}\left(\Omega, \mathbb{R}_{+}\right)$. Soit

$$
A=\left(\begin{array}{cc}
0 & I \\
\Delta & -2 a I
\end{array}\right)
$$

Texte reçu le 15 mai 2001, révisé le 2 avril et le 19 septembre 2002

Moez Khenissi, Faculté des Sciences de Gabès, Département de Mathématiques, route de Medenine, 6029 Gabès (Tunisie) • E-mail : Moez.Khenissi@fsg.rnu.tn

Classification mathématique par sujets (2000). — 35L05, 35S15, 35A07.

Mots clefs. - Équation des ondes, stabilisation, résolvante, scattering. 
l'opérateur non borné défini sur l'espace de Hilbert

$$
H=\widetilde{H_{0}^{1}}(\Omega) \times L^{2}(\Omega)
$$

où $\widetilde{H_{0}^{1}}(\Omega)$ est le complété Hilbertien de $C_{0}^{\infty}(\Omega)$ pour la norme $\|u\|_{1}^{2}=\int_{\Omega}|\nabla u|^{2}$. Il a pour domaine

$$
D(A)=\left\{\left(u_{0}, u_{1}\right) \in H ; \Delta u_{0} \in L^{2}(\Omega), u_{1} \in \widetilde{H_{0}^{1}}(\Omega)\right\} .
$$

Le problème d'évolution

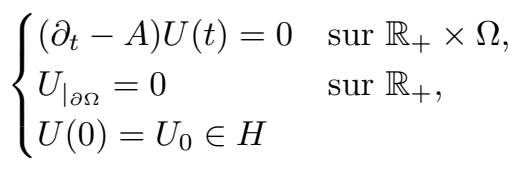

possède une unique solution $U(t)=\mathrm{e}^{t A} U_{0} \in C^{0}\left(\mathbb{R}_{+}, H\right)$, obtenue en appliquant par exemple le théorème de Hille-Yoshida. Pour $R>0, U_{0}$ à support dans $B(0, R)$, on définit son énergie locale à l'instant $t$ dans $\Omega_{R}=B_{R} \cap \Omega$ par

$$
E_{R}(U, t)=\int_{\Omega_{R}}\left[\left|\nabla u_{0}\right|^{2}(t)+\left|u_{1}\right|^{2}(t)\right] \mathrm{d} x .
$$

Elle est bornée par l'énergie initiale.

Dans le cas où $a(x) \equiv 0$, on sait depuis les travaux de P.D. Lax et R.S. Phillips [6] (voir aussi [7], [8]) que l'énergie locale de la solution de l'équation des ondes tend vers 0 quand le temps tend vers l'infini. Puis R. Melrose [10] (voir aussi Morawetz [11]) montre que si l'obstacle est non captif, cette convergence est exponentielle en dimension impaire d'espace et polynômiale en dimension paire par rapport à l'énergie initiale. Dans le cas captif, on sait depuis Ralston [12] qu'il n'existe pas de taux uniforme de décroissance par rapport à l'énergie initiale. Néanmoins, N. Burq [2] montre que le taux est toujours au moins logarithmique, mais par rapport à une norme plus forte que celle de l'énergie initiale $\left(\|\cdot\|_{D\left(A^{k}\right)}\right.$, avec $\left.k>0\right)$.

Dans ce travail, on suppose que $\omega=\{a(x)>0\}$ vérifie le « contrôle géométrique extérieur » au-dessus de $B_{R}$ (C.G.E.), i.e. il existe $T>0$ tel que tout rayon bicaractéristique généralisé $\gamma$ issu d'un point de $T^{*}\left(\mathbb{R}_{+} \times \Omega_{R}\right)$ vérifie l'une des deux conditions suivantes :

a) $\gamma$ quitte $\mathbb{R}_{+} \times \Omega_{R}$ avant l'instant $T$, ou

b) $\gamma$ rencontre la zone $\mathbb{R}_{+} \times \omega$ entre les instants 0 et $T$.

Dans [1], on montre, en dimension impaire d'espace, sous l'hypothèse du « contrôle géométrique extérieur », qu'on a une décroissance exponentielle par rapport à l'énergie intiale, en étudiant le semi-groupe $Z(t)$ de Lax et Phillips adapté au problème.

Dans le présent travail, on se propose d'étudier en premier lieu les pôles de diffusion de l'équation des ondes (1.1). Plus précisément, soit $R(\lambda)$, la résolvante

TOME $131-2003-\mathrm{N}^{\mathrm{O}} 2$ 
sortante du laplacien perturbé avec condition de Dirichlet, holomorphe dans $\{\operatorname{Im} \lambda<0\}$, définie de la manière suivante :

Soit $u(t)$ le propagateur des ondes

$$
\left\{\begin{array}{l}
\left(\partial_{t}^{2}-\Delta+2 a(x) \partial_{t}\right) u(t) f=0 \quad \text { dans } \mathbb{R}_{+} \times \Omega, \\
u(t) f_{\mid \partial \Omega}=0, \quad \forall t \in \mathbb{R}_{+}, \\
u(0) f=0, \\
\partial_{t} u(0) f=f \in C_{0}^{\infty}
\end{array}\right.
$$

qu'on étend comme opérateur de $L^{2}(\Omega)$ dans $H_{0}^{1}(\Omega)$. L'opérateur $R(\lambda)$ est défini par la relation :

$$
R(\lambda)=\int_{0}^{+\infty} \mathrm{e}^{-i \lambda t} u(t) \mathrm{d} t, \quad \text { pour } \quad\{\operatorname{Im} \lambda<0\} .
$$

Comme l'opérateur $u(t)$ est une contraction de $L^{2}(\Omega)$ dans $\widetilde{H_{0}^{1}}(\Omega)$, il est clair que cette relation définit une famille d'opérateurs bornés de $L^{2}(\Omega)$ dans $\widetilde{H_{0}^{1}}(\Omega)$, holomorphe dans $\{\operatorname{Im} \lambda<0\}$.

Soit $\chi \in C_{0}^{\infty}\left(\mathbb{R}^{d}\right), \chi=1$ sur $B_{R}$. La résolvante sortante tronquée

$$
R_{\chi}(\lambda)=\chi R(\lambda) \chi
$$

considérée comme opérateur de $L^{2}(\Omega)$ dans $H_{0}^{1}(\Omega)$, holomorphe dans $\{\operatorname{Im} \lambda<0\}$, s'étend en un opérateur méromorphe (perturbation compacte [8], voir aussi [16]) dans $\mathbb{C}$ en dimension impaire et dans le revêtement simplement connexe de $\mathbb{C}^{*}$ (surface de Riemann du logarithme) si la dimension est paire.

Dans la première partie, on va localiser les pôles de ce prolongement, qu'on appelle pôles de diffusion. Ces pôles sont aussi les points pour les quels il existe une solution sortante non triviale de l'équation

$$
\left(\Delta+\lambda^{2}-i 2 a(x) \lambda\right) u=0, \quad u_{\mid \partial \Omega}=0 .
$$

Ce sont également (en dimension impaire d'espace) les valeurs propres du générateur infinitésimal du semi-groupe $Z(t)$ de Lax-Phillips.

Pour cela, notons $D(0)=\inf \{\operatorname{Im} \lambda, \lambda$ pôle de $R(\lambda)\}$ et

$$
\widetilde{a}(x)=\left\{\begin{array}{l}
a(x) \text { sur } B_{R}, \\
+\infty \text { sur } B_{R}^{c} .
\end{array}\right.
$$

Pour tout $\rho_{0}=\left(x_{0}, \zeta_{0}\right) \in T^{*}(\Omega)$, avec $\left|\zeta_{0}\right|=1$ (et $\zeta_{0}$ appartenant au demiespace fermé défini par $\bar{\Omega}$ si $x_{0} \in \partial \Omega$ ) il existe une unique géodésique généralisée, $s \mapsto x\left(s, \rho_{0}\right)$ de $\bar{\Omega}$ issue de $\rho_{0}$, i.e. vérifiant $x\left(0, \rho_{0}\right)=x_{0}, \lim _{s \rightarrow 0^{+}} x\left(s, \rho_{0}\right) / s=\zeta_{0}$, parcourue à vitesse 1 . Pour $t>0$, on pose

$$
C(t)=\inf _{\rho_{0}} \frac{1}{t} \int_{0}^{t} \tilde{a}\left(x\left(s, \rho_{0}\right)\right) \mathrm{d} s .
$$

BULLETIN DE LA SOCIÉtÉ MATHÉMATIQUE DE FRANCE 
C'est une fonction de $t$, continue, positive, qui vérifie

$$
t C(t)+s C(s) \leq(t+s) C(s+t) .
$$

On note

$$
C(\infty)=\lim _{t \rightarrow+\infty} C(t)
$$

(qui existe car $t C(t)$ est sous-additive [9], et elle peut être infinie). Cette limite est indépendante de $R$, et on a $C(t) \leq C(\infty)$ pour tout $t \geq 0$. Si $\Omega$ est non captif, $C(\infty)=+\infty$, sinon $C(t)$ est majorée par $\|a\|_{\infty}$. La limite $C(\infty)$ ne fait intervenir que la moyenne de $a(x)$ sur les géodésiques captées et sous l'hypothèse du C.G.E., on a $C(\infty)>0$. On a le théorème de localisation suivant.

ThÉORÈme 1.0.1. - Pour tout $\delta<C(\infty)$, il existe $\lambda_{0}>0$ tel que la résolvante sortante tronquée $R_{\chi}(\lambda)$ se prolonge d'une manière holomorphe dans la région

$$
\left\{\lambda \in \mathbb{C}, \operatorname{Im} \lambda \leq \delta,|\operatorname{Re} \lambda| \geq \lambda_{0}\right\} .
$$

Plus précisément, il existe $c>0$ tel que pour toute $f \in L^{2}$, Supp $f \subset B_{R}$ et pour toute $\lambda$ appartenant à la région précédente, on a

$$
\|\nabla R(\lambda) f\|_{L_{R}^{2}}^{2}+\|\lambda R(\lambda) f\|_{L_{R}^{2}}^{2} \leq c\|f\|_{L^{2}}^{2} .
$$

Dans la deuxième partie de ce travail, on déduit que la décroissance de l'énergie locale est exponentielle en dimension impaire et en $1 / t^{d}$ en dimension paire c'est-à-dire, on démontre le théorème :

THÉORÈme 1.0.2. — Sous l'hypothèse du C.G.E. au-dessus de $B_{R}$, pour tout $\delta<\alpha=2 \min (D(0), C(\infty))$, il existe $c>0$ tel que pour tout $f \in H_{R}$, on a

$$
\begin{aligned}
& \left.E_{R}(u(t) f) \leq c \mathrm{e}^{-\delta t} E(u(0) f)\right) \text {, si d est impaire } \\
& \left.E_{R}(u(t) f) \leq \frac{c}{t^{d}} E(u(0) f)\right) \text {, si d est paire }
\end{aligned}
$$

où u est la première composante de la solution de (1.1) pour la donnée intiale $U_{0}=f$.

Soit $\beta$ le meilleur taux de décroissance exponentielle en dimension impaire, défini par

$$
\begin{aligned}
\beta=\sup \{\delta>0 ; \exists c>0, \forall f \in H, \operatorname{Supp} f & \subset B_{R}, \\
& \left.E_{R}(U(t) f) \leq c \mathrm{e}^{-\delta t} E(U(0) f), \forall t \geq 0\right\} .
\end{aligned}
$$

Dans la troisième partie, on calcule $\beta$, en fonction des pôles de $R(\lambda)$ et des moyennes de $\widetilde{a}(x)$ sur les géodésiques de $\Omega$. Cela fait l'objet du théorème :

THÉORÈME 1.0.3. - On a

$$
\beta=2 \min (D(0), C(\infty)) .
$$

TOME $131-2003-\mathrm{N}^{\mathrm{O}} 2$ 


\section{Localisation des pôles de la résolvante}

On commence par démontrer que la résolvante sortante $R_{\chi}(\lambda)$ considérée comme opérateur de $L^{2}(\Omega)$ dans $H_{0}^{1}(\Omega)$ est méromorphe dans $\mathbb{C}$ en dimension impaire et dans la surface de Riemann du $l o g$ en dimension paire d'espace. Ce résultat est classique pour les opérateurs autoadjoints et il reste vrai avec une perturbation compacte [5], [15]. Dans notre cas, on adapte la démonstration de Lax et Phillips [8]. (En realité la démonstration en dimension impaire d'espace est plus simple, en fait elle se déduit de la compacité de $Z(2 R)(\lambda-B)^{-1}$ où $B$ est le générateur infinitésimal du semi-groupe $Z(t)$ de Lax et Phillips [6].)

On rappelle que pour toute $f$ dans $L^{2}(\Omega)$ à support dans $B_{R}, R(\lambda) f$ est l'unique solution sortante du problème nonhomogène suivant :

$$
\left(-\Delta-\lambda^{2}+i 2 \lambda a(x)\right) u=f, \quad u_{\mid \partial \Omega}=0
$$

que l'on peut voir comme perturbation du problème dans l'espace libre :

$$
\left(-\Delta-\lambda^{2}\right) w=g \text { sur } \mathbb{R}^{d}, \quad g \text { dans } L^{2}\left(\Omega_{R}\right)
$$

où $w=R_{0}(\lambda) g$, avec $R_{0}(\lambda)$ la résolvante sortante libre.

On rappelle que

$$
R_{0}(\lambda) g=\int \gamma_{+}(|x-y|, \lambda) g(y) \mathrm{d} y
$$

où

- $\gamma_{+}(r, \lambda)=\mathrm{e}^{-i \lambda r} P_{d}(r, \lambda), P_{d}$ est un polynôme, en dimension impaire d'espace et

- $\gamma_{+}(r, \lambda)=-\frac{i}{4}\left(\frac{\lambda}{2 \pi r}\right)^{\frac{1}{2} n-1} H^{(2)}(\lambda r)$ en dimension paire, où $H^{(2)}$ est la fonction Hankel; on peut noter que $\gamma_{+}$est asymtotiquement [8], [2] comme

$$
r^{-\frac{1}{2}(n-1)} \exp (-i \lambda r) \text {. }
$$

On pose

$$
u=w-\beta h
$$

où

$$
\begin{aligned}
\Delta h+\xi^{2} h-i \xi a(x) h & =0 \text { sur } \Omega_{R}, \\
h & =w \operatorname{sur} \partial \Omega, \\
h & =0 \operatorname{sur}\{|x|=R\}
\end{aligned}
$$

et $\beta \in C_{0}^{\infty}$ valant 1 près $\partial \Omega$ et sur un voisinage de support de $a(x)$ et à support dans $B_{R}, \xi$ étant choisi et fixé suivant la discussion. Ainsi $w$ est complètement déterminé par $g$ et $h$ est complètement determiné par $w$. 
Le problème est de déterminer la fonction $g$ pour laquelle $u$ vérifie (2.1), ainsi

$$
\begin{aligned}
f & =\left(-\Delta-\lambda^{2}+i 2 \lambda a(x)\right) u \\
& =\left(-\Delta-\lambda^{2}+i 2 \lambda a(x)\right) w-\left(-\Delta-\lambda^{2}+i 2 \lambda a(x)\right) \beta h \\
& =g+i 2 \lambda a(x) w+(\Delta \beta) h+2 \nabla \beta \nabla h+\left[\lambda^{2}-\xi^{2}+2 i a(x)(\xi-\lambda)\right] \beta h \\
& =\left(I-T_{\lambda}\right) g
\end{aligned}
$$

où

$$
T_{\lambda} g=-i 2 \lambda a(x) w-(\Delta \beta) h-2 \nabla \beta \nabla h-\left[\lambda^{2}-\xi^{2}+2 i a(x)(\xi-\lambda)\right] \beta h .
$$

LEMME 2.0.1. - On a :

a) $T_{\lambda}$ est un opérateur compact sur $L_{2}\left(\Omega_{R}\right)$ pour tout $\lambda \in \mathbb{C} \backslash\{0\}$.

b) $T_{\lambda}$ est une fonction holomorphe en $\lambda$ dans $\mathbb{C}$ en dimension impaire et dans la surface de Riemann du logarithme en dimension paire.

Preuve de a). — Soit $H^{k}\left(\Omega_{R}\right)$ l'espace de Sobolev des fonctions définies sur $\Omega_{R}$ qui sont $k$ fois dérivables, ayant comme norme

$$
\|h\|_{k}^{\prime}=\left\{\sum_{|\alpha| \leq k} \int_{\Omega_{R}}\left|\partial^{\alpha} h\right|^{2} \mathrm{~d} x\right\}^{\frac{1}{2}} .
$$

À partir de (2.3) et de la théorie des intégrales oscillantes on peut voir que

$$
\|\beta w\|_{2}^{\prime} \leq C_{\lambda}\|g\|_{0}^{\prime},
$$

où $C_{\lambda}$ est uniformement borné sur tout compact de la surface de Riemann du logarithme en dimension paire d'espace et de $\mathbb{C}$ en dimension impaire d'espace.

Maintenant, $v=h-\beta w$ satisfait l'équation

$$
\begin{aligned}
\Delta v+\xi^{2} v-i \xi a(x) v & =-\Delta(\beta w)-\xi^{2} \beta w+i \xi a(x) \beta w \text { sur } \Omega_{R} \\
v & =0 \text { sur } \partial \Omega_{R} .
\end{aligned}
$$

On déduit par un argument d'ellipticité que

$$
\|v\|_{2}^{\prime} \leq C\|\beta w\|_{2}^{\prime} \text {. }
$$

En combinant avec (2.6), on obtient

$$
\|h\|_{2}^{\prime} \leq C C_{\lambda}\|g\|_{0}^{\prime} .
$$

Comme $T_{\lambda} g$ ne contient que des dérivations de premier ordre de $h$,

$$
\left\|T_{\lambda} g\right\|_{1}^{\prime} \leq C\|h\|_{2}^{\prime} \leq C_{\lambda}^{\prime}\|g\|_{0}^{\prime},
$$

et d'après le théorème de compacité de Rellich, on déduit que $T_{\lambda}$ est un opérateur compact sur $L_{2}\left(\Omega_{R}\right)$.

TOME $131-2003-\mathrm{N}^{\mathrm{O}} 2$ 
Preuve de b). - Il est connu que $R_{0}(\lambda)$, considéré comme opérateur de $L_{2}\left(\Omega_{R}\right)$ dans l'espace de Sobolev $H^{2}\left(\Omega_{R}\right)$, est holomorphe sur $\mathbb{C}$ en dimension impaire d'espace et dans la surface de Riemann du logarithme en dimension paire. On peut voir à partir de la relation $h=v+\beta w$ et (2.10) que $T_{\lambda}$ est holomorphe, ainsi on achève la preuve du lemme 2.0.1.

On définit l'opérateur $Q$ par $Q: w \rightarrow \beta h$ et la résolvante sortante $R(\lambda)$ par

$$
u=R(\lambda) f=(I-Q) R_{0}(\lambda)\left(I-T_{\lambda}\right)^{-1} f
$$

de $L_{2}\left(\Omega_{R}\right)$ dans $H^{2}\left(\Omega_{R}\right)$. Toujours d'après la relation $h=v+\beta w$ et $(2.10)$, on voit que $Q$ est un opérateur borné sur $H^{2}\left(\Omega_{R}\right)$. Le lemme 2.0.1 implique que $\left(I-T_{\lambda}\right)^{-1}$ est méromorphe sur le domaine d'holomorphie de $T_{\lambda}$. Comme $u$ est déterminé par $R_{0}(\lambda)\left(I-T_{\lambda}\right)^{-1}$ à l'extérieur de la boule $\{|x|<R\}$, on peut bien prendre l'espace image de $R(\lambda)$ dans $H^{2}\left(\Omega_{R^{\prime}}\right)$ pour tout $R^{\prime}>R$.

Pour conclure, l'opérateur $R(\lambda)$ considéré comme fonction en $\lambda$ (de $L_{2}\left(\Omega_{R}\right)$ dans $H^{2}\left(\Omega_{R^{\prime}}\right)$ avec $R^{\prime}>R$ ) est méromorphe sur $\mathbb{C}$ en dimension impaire d'espace et dans la surface de Riemann du logarithme en dimension paire.

2.1. Études des hautes fréquences. - Cette section est consacrée à la preuve du théorème 1.0.1.

Comme $R_{\chi}(\lambda)$, considéré comme opérateur de $L^{2}(\Omega)$ dans $H_{0}^{1}(\Omega)$, est méromorphe dans $\mathbb{C}$ en dimension impaire et dans la surface de Riemann du logarithme, holomorphe sur $\{\operatorname{Im} \lambda<0\}$ et que $c, \delta$ et $\lambda_{0}$ ne dépendent pas de $\lambda$, il suffit de montrer l'inégalité (1.7) pour $\lambda$ non pôle de $R(\lambda)$ et appartenant à la région $\left\{\lambda \in \mathbb{C}, \operatorname{Im} \lambda \leq \delta,|\operatorname{Re} \lambda| \geq \lambda_{0}\right\}$; par suite, on déduit que $R(\lambda)$ n'a pas des pôles dans cette région et qu'elle vérifie (1.7). De plus, comme $R_{\chi}(-\bar{\lambda})=\overline{R_{\chi}(\lambda)}$, on limite notre étude à l'ensemble $\operatorname{Re} \lambda>0$. Enfin il est clair qu'il n'y a rien à démontrer lorsque $C(\infty)=0$ (ce cas correspond à un ouvert $\Omega$ captif et à l'hypothèse C.G.E. non vérifiée). On s'intéresse donc aux réels $\delta>0$. La preuve est basée sur un raisonnement par l'absurde.

On suppose qu'il existe $\delta<C(\infty)$ tel que pour tout $\lambda_{0}$ et pour tout $c$ (en particulier pour $\lambda_{0}=c=n \in \mathbb{N}$ ), il existe $f_{n} \in L^{2}$ et $\operatorname{Supp} f_{n} \subset B_{R}$, tel que $\operatorname{Re} \lambda_{n} \geq n, 0 \leq \operatorname{Im} \lambda_{n} \leq \delta$ vérifiant

$$
\left\|\nabla R\left(\lambda_{n}\right) f_{n}\right\|_{L_{R}^{2}}^{2}+\left\|\lambda_{n} R\left(\lambda_{n}\right) f_{n}\right\|_{L_{R}^{2}}^{2} \geq n\left\|f_{n}\right\|_{L^{2}}^{2}
$$

On note $u_{n}=R\left(\lambda_{n}\right) f_{n}$, on normalise par $\left\|\nabla u_{n}\right\|_{L_{R}^{2}}^{2}+\left\|\lambda_{n} u_{n}\right\|_{L_{R}^{2}}^{2}<\infty$. La suite $\operatorname{Im} \lambda_{n}$ est bornée, elle admet une sous-suite convergente. On obtient ainsi modulo cette sous-suite

$$
\begin{aligned}
& \quad\left\{\begin{array}{l}
-\Delta u_{n}-\lambda_{n}^{2} u_{n}+2 i a(x) \lambda_{n} u_{n}=f_{n} \text { dans } \Omega \\
u_{n \mid \partial \Omega}=0, \quad u_{n} \text { sortante }
\end{array}\right. \\
& \left\|\nabla u_{n}\right\|_{L_{R}^{2}}^{2}+\left\|\lambda_{n} u_{n}\right\|_{L_{R}^{2}}^{2}=1, \quad\left\|f_{n}\right\|_{L_{R}^{2}} \rightarrow 0 \text { et }\left\|u_{n}\right\|_{L_{R}^{2}}^{2} \rightarrow 0
\end{aligned}
$$

BULletin DE LA SOCIÉtÉ MATHÉMATIQUE DE FRANCE 
et $h_{n}=1 /\left(\operatorname{Re} \lambda_{n}\right) \rightarrow 0, \operatorname{Im} \lambda_{n} \rightarrow \delta_{0} \leq \delta$.

On examine à présent la suite $u_{n}$.

LEMME 2.1.1. - On a $u_{n} \rightarrow 0$ dans $H_{\mathrm{loc}}^{1}(\Omega)$ et $\lambda_{n} u_{n} \rightarrow 0$ dans $L_{\mathrm{loc}}^{2}(\Omega)$.

Démonstration. - On a (2.12) implique que $u_{n} \rightarrow 0$ dans $H_{R}^{1}$ et de plus

$$
\lambda_{n} u_{n}=-\frac{1}{\lambda_{n}} \Delta u_{n}+2 i a(x) u_{n}-\frac{1}{\lambda_{n}} f_{n} .
$$

En effectuant le produit scalaire $L^{2}$ avec $\psi \in C_{0}^{\infty}(\Omega)$, on voit que $\lambda_{n} u_{n} \rightarrow 0$ dans $L_{R}^{2}$.

Soit $\chi \in C_{0}^{\infty}$ valant 1 près de $\partial \Omega$ et sur un voisinage du support de $a(x)$ et à support dans $B_{R}$. On pose $w_{n}=(1-\chi) u_{n}$. On vérifie que

$$
-\left(\lambda_{n}^{2}+\Delta\right) w_{n}=[\Delta, \chi] u_{n}-(1-\chi) f_{n} \quad \text { dans } \mathbb{R}^{d}
$$

donc $w_{n}=R_{0}\left(\lambda_{n}\right) g_{n}$ où $R_{0}$ est la résolvante libre du laplacien et

$$
g_{n}=[\Delta, \chi] u_{n}-(1-\chi) f_{n},
$$

bornée dans $L^{2}\left(\mathbb{R}^{d}\right)$ et à support dans $B_{R}$. On a

$$
R_{0}\left(\lambda_{n}\right) g_{n}=\int_{0}^{+\infty} \mathrm{e}^{-i \lambda_{n} t} u_{0}(t) g_{n} \mathrm{~d} t,
$$

où $u_{0}(t)$ est le propagateur libre. Ainsi par intégration par parties et en remarquant que l'énergie locale tend vers 0 en $+\infty$,

$$
\lambda_{n} R_{0}\left(\lambda_{n}\right) g_{n}=\int_{0}^{+\infty} \mathrm{e}^{-i \lambda_{n} t} \partial_{t} u_{0}(t) g_{n} \mathrm{~d} t .
$$

Comme $\operatorname{Im} \lambda_{n} \leq \delta$, pour $\operatorname{Re} \lambda_{n}$ assez grand, pour $R^{\prime}>R$, on a

$$
\left\|\nabla w_{n}\right\|_{B_{R^{\prime}}}^{2}+\left\|\lambda_{n} w_{n}\right\|_{B_{R^{\prime}}}^{2} \leq c\left\|g_{n}\right\|^{2} .
$$

On peut démontrer cette inégalité en dimension impaire d'espace d'une manière simple, en se basant sur le principe de Huyghens $\left(E_{t-R}(u(t) f)=0\right.$ pour tout $t \geq R$ et tout $f \in H_{R}$ ) et en dimension paire sur la méthode de la phase stationaire pour $\operatorname{Re} \lambda_{n} \rightarrow+\infty$ dans l'expression intégrale (2.6) (voir [2]). On déduit que $w_{n}$ est bornée dans $H_{\text {loc }}^{1}\left(B_{R}^{c}\right)$ et que $\left\|w_{n}\right\|_{L_{\text {loc }}^{2}} \rightarrow 0$. Ainsi $u_{n} \rightarrow 0$ dans $H_{\text {loc }}^{1}$ et (2.13) implique que $\lambda_{n} u_{n} \rightarrow 0$ dans $L_{\text {loc }}^{2}$.

On pose

$$
v_{n}(t, x)=\mathrm{e}^{-i t / h_{n}} u_{n}(x) .
$$

On déduit facilement du lemme 2.1.1 que $v_{n} \rightarrow 0$ dans $H_{\text {loc }}^{1}(\mathbb{R} \times \Omega)$. On peut ainsi lui associer une mesure de défaut microlocale $\mu$ dans $H_{\text {loc }}^{1}(\mathbb{R} \times \Omega)$ (voir [9], nous renvoyons le lecteur aux articles originaux [4], [13] de P. Gérard 
et L. Tartar pour une exposition de la théorie des $H$-mesures, ou mesures de défauts microlocales). La suite $v_{n}$ vérifie l'équation des ondes

$$
\left\{\begin{array}{l}
\square v_{n}-\left(2 a(x)-2 \delta_{0}\right) \partial_{t} v_{n}=\mathrm{e}^{-i t / h_{n}} \tilde{f}_{n}, \\
v_{n \mid \partial \Omega}=0
\end{array}\right.
$$

avec $\tilde{f}_{n}=f_{n}+\operatorname{Im} \lambda_{n}\left(2 a(x)-\operatorname{Im} \lambda_{n}\right) u_{n}+2 i \operatorname{Re} \lambda_{n}\left(\operatorname{Im} \lambda_{n}-\delta_{0}\right) u_{n}$. On vérifie que $\left\|\tilde{f}_{n}\right\|_{L_{\text {loc }}^{2}}$ tend vers zéro car

$$
\begin{gathered}
\left\|f_{n}\right\|_{L^{2}} \rightarrow 0, \quad\left\|\operatorname{Im} \lambda_{n}\left(2 a(x)-\operatorname{Im} \lambda_{n}\right) u_{n}\right\|_{L_{\mathrm{loc}}^{2}}^{2} \leq c\left\|u_{n}\right\|_{L_{\mathrm{loc}}^{2}}^{2} \rightarrow 0, \\
\left\|\operatorname{Re} \lambda_{n}\left(\operatorname{Im} \lambda_{n}-\delta_{0}\right) u_{n}\right\|_{L_{\mathrm{loc}}^{2}}^{2} \leq c\left|\operatorname{Im} \lambda_{n}-\delta_{0}\right| \cdot\left\|\lambda_{n} u_{n}\right\|_{L_{\mathrm{loc}}^{2}} \leq c\left|\operatorname{Im} \lambda_{n}-\delta_{0}\right| \rightarrow 0 .
\end{gathered}
$$

Ainsi $\square v_{n}$ est compact dans $L_{\text {loc }}^{2}(\mathbb{R} \times \Omega)$. On peut donc appliquer le théorème de régularité microlocale pour les mesures de défaut. On a Supp $\mu \subset\left\{|\xi|^{2}=\tau^{2}\right\}$ variété caractéristique de l'opérateur des ondes.

Nous allons montrer de plus le

Lemme 2.1.2. - Pour tout $x \notin B_{R}$, on a

$$
\operatorname{Supp} \mu \subset\left\{(t, x, \tau, \xi) ;|\xi|^{2}=\tau^{2} \text { et } x \cdot \xi<0\right\} .
$$

Démonstration. - Soit $x_{0} \in \Omega,\left|x_{0}\right|>R$ et $\left(\tau_{0}, \xi_{0}\right) \in \mathbb{R}^{+} \times \mathbb{R}^{d}$ tel que $x_{0} \cdot \xi_{0} \geq 0$ et $\left|\xi_{0}\right|^{2}=\tau_{0}^{2}$. Soit $\chi \in C_{0}^{\infty}(\Omega)$, à support dans un voisinage $V_{x_{0}}$ de $x_{0}$ inclus dans $B_{R}^{c}$ et $\varphi \in C_{0}^{\infty}([0, T])$ pour $T>0$. Soit $a(x, \tau, \xi) \in S^{0}$, homogène d'ordre zéro, un symbole de microlocalisation près de $\left(x_{0}, \tau_{0}, \xi_{0}\right)$, valant 1 en ce point. On a

$$
\begin{aligned}
&\left\langle a\left(x, D_{x}, D_{t}\right) \varphi \chi v_{n}, \varphi \chi v_{n}\right\rangle_{H_{x, t}^{1}} \\
& \underset{n \rightarrow \infty}{\longrightarrow} \int_{\Omega \times[0, T] \times S^{d}} a(x, \xi, \tau) \varphi(t) \chi(x) \mathrm{d} \mu(x, t, \xi, \tau) .
\end{aligned}
$$

Pour déduire le résultat du lemme 2.1.2, il suffit de démontrer que

$$
\left\langle a\left(x, D_{x}, D_{t}\right) \varphi \chi \nabla v_{n}, \varphi \chi \nabla v_{n}\right\rangle_{L^{2}}+\left\langle a\left(x, D_{x}, D_{t}\right) \varphi \chi \partial_{t} v_{n}, \varphi \chi \partial_{t} v_{n}\right\rangle_{L^{2}} \longrightarrow 0 .
$$

Comme $u_{n}=w_{n}$ sur $B_{R}^{c}$ et, en outre, remarquant qu'une dérivation en espace de $w_{n}$ ou en temps de $v_{n}$ est essentiellement une multiplication par $\lambda_{n}$, il suffit de montrer que $\lambda_{n} a\left(x, D_{x}, D_{t}\right) \varphi \chi v_{n}$ tend vers 0 , pour la norme uniforme. On a

$$
\begin{aligned}
& a\left(x, D_{x}, D_{t}\right) \varphi \chi v_{n} \\
& \quad=\iint_{y \in V_{x_{0}}} \mathrm{e}^{i(x-y) \xi+i(t-s) \tau} \varphi(s) \chi(y) v_{n}(s, y) a(x, \xi, \tau) \mathrm{d} y \mathrm{~d} s \mathrm{~d} \xi \mathrm{d} \tau .
\end{aligned}
$$

BULLETIN DE LA SOCiÉtÉ MATHÉMATIQUE DE FRANCE 
Remarque 2.1.1. - En tenant compte du fait que $\operatorname{Re} \lambda_{n}$ tend vers $+\infty$, que $\operatorname{Im} \lambda_{n}$ tend vers $\delta_{0}$ et que l'expression de la résolvante libre admet le même comportement asymtotique en dimension impaire qu'en dimension paire d'espace [8], on va se restreindre, pour simplifier, à l'expression de $R_{0}\left(\lambda_{n}\right)$ en dimension impaire [3].

On a

$$
w_{n}(y)=\int_{z \in \operatorname{Supp} g_{n}} \mathrm{e}^{-i \lambda_{n}|y-z|} \mathcal{P}_{d}\left(|y-z|, \lambda_{n}\right) g_{n}(z) \mathrm{d} z
$$

où

$$
\mathcal{P}_{d}(r, \lambda)=c(d)\left(\frac{1}{r} \frac{\partial}{\partial r}\right)^{\frac{1}{2}(d-2)}\left(\frac{\mathrm{e}^{-i \lambda r}}{r}\right) \mathrm{e}^{i \lambda r}=\sum_{\alpha, \beta \in I} c(d) \frac{\lambda^{\alpha}}{r^{\beta}},
$$

$|I|$ ne dépend que de $d$. Donc

$$
a\left(x, D_{x}, D_{t}\right) \varphi \chi v_{n}=c(d) \sum_{\alpha, \beta \in I} J_{\alpha, \beta, n}(x),
$$

avec

$$
\begin{aligned}
J_{\alpha, \beta, n}= & c \iint_{s \in[0, T]} \varphi(s) \mathrm{e}^{i(t-s) \tau-i s / h_{n}} \\
& \times \iint_{y \in V_{x_{0}}, z \in B_{R}} \lambda^{\alpha} \mathrm{e}^{i(x-y) \xi-i / h_{n}|y-z|} \chi(y) a(x, \xi, \tau) \frac{g_{n}(z)}{|y-z|^{\beta}} \mathrm{e}^{\operatorname{Im} \lambda_{n}|y-z|} .
\end{aligned}
$$

Soient les phases

$$
\Gamma_{n}(y)=y \cdot \xi+\frac{1}{h_{n}}|y-z|, \quad \delta(s)=s \tau .
$$

On a

$$
\nabla_{y} \Gamma_{n}=\frac{1}{h_{n}}\left(h_{n} \xi+\frac{(y-z)}{|y-z|}\right)
$$

en vertu de $x \cdot \xi \geq 0$, on peut voir aisément qu'il existe $c_{1}$ et $c_{2}>0$ tels que

$$
\left|\nabla_{y} \Gamma_{n}\right| \geq \frac{c_{1}}{h_{n}} \quad \text { et } \quad\left|\nabla_{y} \Gamma_{n}\right| \geq c_{2}|\xi|
$$

On va intégrer par parties à l'aide des opérateurs

$$
L_{n}\left(\xi, y, \partial_{y}\right)=\frac{\nabla_{y} \Gamma_{n} \cdot \partial_{y}}{-i\left|\nabla_{y} \Gamma_{n}\right|^{2}}, \quad{ }^{t} L_{n}=\partial_{y} \cdot\left(\frac{\nabla_{y} \Gamma_{n}}{i\left|\nabla_{y} \Gamma_{n}\right|^{2}}\right)=\ell_{n}\left(\xi, y, \partial_{y}\right)
$$

et

$$
H={ }^{t} H=1-\partial_{s}^{2} .
$$

Ils vérifient

$$
L_{n}\left(\mathrm{e}^{-i \Gamma_{n}}\right)=\mathrm{e}^{-i \Gamma_{n}} \quad \text { et } \quad H\left(\mathrm{e}^{-i \delta}\right)=\left(1+\tau^{2}\right) \mathrm{e}^{-i \delta} .
$$

TOME $131-2003-\mathrm{N}^{\mathrm{O}} 2$ 
On remarque que $\tilde{\ell_{n}}=O\left(h_{n}|\xi|^{-1}\right)$ (i.e. $\exists c>0$ tel que $\left(1+|\xi|^{2}\right)^{1 / 2} \tilde{\ell_{n}}<c h_{n}$, pour tous $y \in V_{x_{0}}, z \in B_{R}$ et $\left.\xi \in \mathbb{R}^{d}\right)$ où $\tilde{\ell_{n}}$ représente les coefficients de $\ell_{n}$.

En intégrant par parties $N$ fois par $L_{n}$ et $M$ fois par $H$, on obtient

$$
\begin{aligned}
J_{\alpha, \beta, n}= & \left.c \lambda_{n}^{\alpha} \iint \mathrm{e}^{i(t-s) \tau} \frac{1}{\left(1+\tau^{2}\right)^{M}} H^{M}(\varphi(s)) \mathrm{e}^{-i s / h_{n}}\right) \\
& \times \iint \mathrm{e}^{i(x-y) \xi-i / h_{n}|y-z|} a(x, \xi, \tau)\left({ }^{t} L_{n}\right)^{N}\left(\chi(y) \frac{g_{n}(z)}{|y-z|^{\beta}} \mathrm{e}^{\operatorname{Im} \lambda_{n}|y-z|}\right) . \\
= & \left.c \lambda_{n}^{\alpha} \iint \mathrm{e}^{i(t-s) \tau} \frac{1}{\left(1+\tau^{2}\right)^{M}} H^{M}(\varphi(s)) \mathrm{e}^{-i s / h_{n}}\right) \\
& \times \int \mathrm{e}^{i(x-y) \xi} \frac{a(x, \xi, \tau)}{\left(1+|\xi|^{2}\right)^{\frac{1}{2} N}}\left(1+|\xi|^{2}\right)^{\frac{1}{2} N} \int_{z \in B_{R}} \mathrm{e}^{-i / h_{n}|y-z|} \ell_{n}^{N}() .
\end{aligned}
$$

En remarquant que $\left.H^{M}(\varphi(s)) \mathrm{e}^{-i s / h_{n}}\right)$ est en $O\left(h_{n}^{-2 M}\right)$, on obtient

$$
\begin{aligned}
\left|\lambda_{n} J_{\alpha, \beta, n}\right| \leq c\left|\lambda_{n}\right|^{\alpha+1} h_{n}^{-2 M} \int & \frac{1}{\left|1+\tau^{2}\right|^{M}} \frac{|a(x, \xi, \tau)|}{\left(1+|\xi|^{2}\right)^{\frac{1}{2} N}}\left(1+|\xi|^{2}\right)^{\frac{1}{2} N} \\
& \times \int_{z \in B_{R}}\left|\ell_{n}^{N}\left(\frac{\chi(y) g_{n}(z)}{|y-z|^{\beta}} \mathrm{e}^{\operatorname{Im} \lambda_{n}|y-z|}\right)\right| .
\end{aligned}
$$

Comme les coefficients de $\ell$ sont d'ordre -1 en $\xi, \operatorname{Im} \lambda_{n}<\delta_{0}$ et $\chi \in C_{0}^{\infty}$, alors il existe $c \in \mathbb{R}_{+}$tel que

$$
\begin{aligned}
\left(1+|\xi|^{2}\right)^{\frac{1}{2} N} \int_{z \in B_{R}} \mid \ell_{n}^{N}\left(\frac{\chi(y) \mathrm{e}^{\operatorname{Im} \lambda_{n}|y-z|}}{|y-z|^{\beta}}\right) & g_{n}(z) \mid \\
& \leq c h_{n}^{N} \int_{z \in B_{R}}\left|g_{n}(z)\right|^{2} \mathrm{~d} z,
\end{aligned}
$$

quels que soient $y \in V_{x_{0}}, \xi \in \mathbb{R}^{d}$ et $n \in \mathbb{N}$.

Or $g_{n}$ est bornée dans $L^{2}$; ainsi pour tout $x \in V_{x_{0}}$, on a

$$
\begin{aligned}
\left|\lambda_{n} J_{\alpha, \beta, n}\right| & \leq c\left|\lambda_{n}\right|^{\alpha+1} h^{N-2 M} \iint_{\tau, \xi} \frac{1}{\left|1+\tau^{2}\right|^{M}} \cdot \frac{|a(x, \xi, \tau)|}{\left(1+|\xi|^{2}\right)^{\frac{1}{2} N}} \mathrm{~d} \tau \mathrm{d} \xi \\
& \leq c\left|\lambda_{n}\right|^{\alpha+1} h^{N-2 M} \underset{n \rightarrow \infty}{\longrightarrow} 0, \quad \text { pour } N \text { assez grand. }
\end{aligned}
$$

Et donc

$$
\sup _{x \in V_{x_{0}}}\left(\lambda_{n} a\left(x, D_{x}, D_{t}\right) \varphi \chi V_{n}\right) \underset{n \rightarrow \infty}{\longrightarrow} 0 .
$$

On déduit que $\int a(x, \xi, \tau) \varphi \chi \mathrm{d} \mu=0$ et par suite $\mu$ ne charge pas $\left(x_{0}, \xi_{0}, \tau_{0}\right)$.

Remarque 2.1.2. - Le lemme 2.1.2 exprime tout simplement qu'en dehors de $B_{R}$ la mesure $\mu$ est nulle sur les rayons sortants $(x \cdot \xi \geq 0)$. Dans toute la suite, la mesure $\mu$ est définie sur la composante connexe $\tau>0\left(S Z^{+}\right.$, voir [9]). Ainsi les rayons pour lesquels $x \cdot \xi>0$ sont les sortantes dans le futur $(s>0)$. 
Maintenant grâce aux lemmes 2.1.1 et 2.1.2, on va trouver une absurdité en utilisant le théorème de propagation de Lebeau [9]. On rappelle que $v_{n}$ vérifie l'équation des ondes amorties :

$$
\left\{\begin{array}{l}
\square v_{n}-\left(2 a(x)-2 \delta_{0}\right) \partial_{t} v_{n}=\mathrm{e}^{i t / h_{n}} \tilde{f}_{n}, \\
v_{n_{\mid \partial \Omega}}=0
\end{array}\right.
$$

avec $\left\|\mathrm{e}^{i t / h_{n}} \tilde{f}_{n}\right\|_{L_{\text {loc }}^{2}} \rightarrow 0$ lorsque $n$ augmente indéfiniment.

Ainsi, d'après [9], pour tout $\omega$ borélien en $(x, t, \xi, \tau)$ dans $T^{*} B_{R} \times[0, T]$, $T>0$, on a

$$
\mu(G(s) \omega)=\int_{\omega} \exp \left(\int_{0}^{s}\left(2 a(G(\sigma)(x, t, \xi, \tau))-2 \delta_{0}\right) \mathrm{d} \sigma\right) \mathrm{d} \mu(x, t, \xi, \tau)
$$

où $G(s)$ est le flot bicaractéristique généralisé associé à $\square$. On rappelle que $\{G(s)\}$ est un groupe de $C^{0}$-homéomorphismes.

On a Supp $\mu \cap B_{R} \neq \varnothing$, car sinon $\mu=0$ sur $B_{R}$. En appliquant alors le lemme 2.1.2 et le théorème de propagation des mesures (P. Gérard [4]), [9], on aurait $\mu=0$ sur $B_{R^{\prime}}$ où $R^{\prime}>R$ et donc $v_{n} \rightarrow 0$ dans $H_{t, x}^{1}\left(B_{R} \times[0, T]\right)$. Ainsi

$$
u_{n} \rightarrow 0 \text { dans } H_{R}^{1}, \quad \text { et } \quad \lambda_{n} u_{n} \rightarrow 0 \text { dans } L_{R}^{2},
$$

ce qui est absurde car $\left\|\nabla u_{n}\right\|_{L_{R}^{2}}^{2}+\left\|\lambda_{n} u_{n}\right\|_{L_{R}^{2}}^{2}=1$.

Soit donc $\omega$ un borélien de $T^{*}\left(B_{R}\right) \times[0,1]$ tel que $\mu(\omega) \neq 0$.

On écrit $\omega=\omega_{1} \cup \omega_{2}$ où $\omega_{1}$ et $\omega_{2}$ sont les deux boréliens définis par

$\omega_{1}=\left\{\rho \in \omega ; \exists s, G(s) \rho \notin B_{R}\right\}, \quad \omega_{2}=\left\{\rho \in \omega ; \forall s \geq 0, G(s) \rho \in B_{R}\right\}$.

On a $\mu(\omega)=\mu\left(\omega_{1}\right)+\mu\left(\omega_{2}\right)$. Or $\mu\left(\omega_{1}\right)=0$, car si $\rho \in \omega_{1}$, il existe $s$ tel que $G(s) \rho \notin B_{R}$; donc $G(s) \rho$ est sortante et en appliquant le lemme 2.1.2, $\mu\left(\omega_{1}\right)=0$. Ainsi $\mu(\omega)=\mu\left(\omega_{2}\right)$.

On remarque que si $\Omega$ est non captif, $\omega=\omega_{1}$ implique $\mu(\omega)=0$, ce qui est absurde. Donc il reste le cas où $\Omega$ est captif avec l'hypothèse de C.G.E. au-dessus de $B_{R}$.

On a $G(s) \omega_{2} \subset T^{*}\left(B_{R}\right) \times[s, s+1]$ quel que soit $s \geq 0$, donc

$$
\mu\left(G(s) \omega_{2}\right) \leq \mu\left(B_{R} \times[s, s+1]\right) \leq\left\|v_{n}\right\|_{H_{R,[0,1]}^{1}}^{2} \leq 1 .
$$

On rappelle que $C(s)=\inf _{\rho} s^{-1} \int_{0}^{s} \tilde{a}(G(\tau) \rho) \mathrm{d} \tau$.

En remarquant que toutes les bicaractéristiques issues de $\omega_{2}$ sont captives, on obtient d'après (2.26)

$$
\mu\left(G(s) \omega_{2}\right) \geq \mathrm{e}^{\left[2 C(s)-2 \delta_{0}\right] s} \mu\left(\omega_{2}\right) .
$$

On a $\delta_{0}<C(\infty)$, donc il existe $\varepsilon>0$ tel que $\delta_{0} \leq C(\infty)-\varepsilon$.

TOME $131-2003-\mathrm{N}^{\mathrm{O}} 2$ 
Soit $s_{0}>0$ tel que $C(\infty)-C\left(s_{0}\right) \geq \frac{1}{2} \varepsilon$; pour tout $s \geq s_{0}$, on a

$$
\begin{aligned}
\mu\left(G(s) \omega_{2}\right) & \geq \mathrm{e}^{\left[2 C(s)-2 C(\infty)+2 C(\infty)-2 \delta_{0}\right] s} \mu(\omega) \\
& \geq \mathrm{e}^{(-\varepsilon+2 \varepsilon) s} \mu(\omega) \\
& \geq \mathrm{e}^{\varepsilon s} \mu(\omega) .
\end{aligned}
$$

Pour $s$ assez grand on obtient $\mu\left(G(s) \omega_{2}\right)>1$ (car $\left.\mu(\omega) \neq 0\right)$, ce qui contredit (2.27).

2.2. Basses fréquences. - Le comportement de $R_{\chi}(\lambda)$ en basses fréquences (près de l'origine) dépend de la parité de la dimension d'espace [14]. En fait, en dimension paire, 0 présente une singularité de type branche logarithmique [16], [14] et en dimension impaire l'ensemble des pôles de $R_{\chi}(\lambda)$ n'admet pas de points d'accummulation [6] et il ne coupe pas l'axe réel. Donc sur l'ensemble $\{|\lambda|<c\}$, les pôles de $R_{\chi}(\lambda)$ sont en nombre fini et donc isolés. Plus précisement, en tenant compte de la proposition 3.1 de [16], [14] $\left(R_{\chi}(\lambda)\right.$ considéré comme opérateur de $\left.L^{2}(\Omega) \rightarrow L^{2}(\Omega)\right)$, on a

LEMmE 2.2.1. - Si $d \geq 2$ est paire, pour $\lambda \rightarrow 0$ et $\left|\arg \lambda+\frac{1}{2} \pi\right| \leq \pi$, on a

$$
R_{\chi}(\lambda)=\mathcal{M}_{d} \lambda^{d-2} \log \lambda+\mathcal{F}_{d}(\lambda)+O\left(|\lambda|^{d-2}\right),
$$

où $\operatorname{rang} \mathcal{M}_{d}=1$ et $\mathcal{F}_{d}$ est un polynôme de degré $\leq d-3$ si $d \geq 4$ et $\mathcal{F}_{2} \equiv 0$.

Démonstration. - Soit $\widetilde{R}(\lambda): L_{\text {comp }}^{2}(\Omega) \rightarrow L_{\text {comp }}^{2}(\Omega)$ l'opérateur défini par

$\widetilde{R}(\lambda) f$ est l'unique solution de $\left(-\Delta-\lambda^{2}\right) u=f, u_{\mid \partial \Omega}=0$, u sortante.

Soit alors $f \in L^{2}(\Omega)$ est à support dans $B_{R} ; a(x)$ étant aussi à support compact dans $B_{R}$, la solution $u$ du système

$$
\left(-\Delta-\lambda^{2}+i \lambda a(x)\right) u=f+i \lambda a(x) u, \quad u_{\mid \partial \Omega}=0, \quad u \text { sortante }
$$

s'écrit comme

$$
\begin{aligned}
\widetilde{R}(\lambda) f & =u=R(\lambda)(f+i \lambda a(x) u) \\
& =R(\lambda)(f+i \lambda a(x) \widetilde{R}(\lambda) f) \\
& =R(\lambda)[I+i \lambda a(x) \widetilde{R}(\lambda)] f .
\end{aligned}
$$

Ainsi pour toute $f \in L^{2}$ à support dans $B_{R}$,

$$
\widetilde{R}_{\chi}(\lambda) f=R_{\chi}(\lambda)\left[I+i \lambda a(x) \widetilde{R}_{\chi}(\lambda)\right] f .
$$

En tenant compte de la proposition $3.1 \mathrm{de}$ Vodev [16], on a $i \lambda a(x) \widetilde{R}_{\chi}(\lambda) \rightarrow 0$ quand $\lambda \rightarrow 0$. Donc $I+i \lambda a(x) \widetilde{R}_{\chi}(\lambda)$ est inversible et on peut voir aisément que $R_{\chi}(\lambda)$ et $\widetilde{R}_{\chi}(\lambda)$ ont le même comportement près de 0 . 
Remarque 2.2.1. - Il est clair qu'en dimension paire, le lemme 2.2.1 donne, modulo une fonction analytique en 0 ,

$$
\lambda R_{\chi}(\lambda)=\mathcal{M}_{d} \lambda^{d-1} \log \lambda+O\left(|\lambda|^{d-1}\right), \quad \lambda \rightarrow 0 .
$$

De plus, on peut voir facilement de la preuve du lemme que la même conclusion est établie pour $\chi \partial_{x_{j}} R \chi(\lambda), j=1, \ldots, d$.

\section{Décroissance de l'énergie locale}

Cette section est consacrée à la preuve du théorème 1.0.2.

Comme dans Morawetz [11], on considère une fonction $\varphi(t) \in C^{\infty}(\mathbb{R})$ telle que

$$
\varphi(t)=\left\{\begin{array}{l}
0 \text { pour } t \leq 1 \\
1 \text { pour } t \geq 2
\end{array}\right.
$$

Soit $V(t)=\varphi(t) U(t)$ où $U(t)=\mathrm{e}^{i t G}$ et $G=-i A$. La transformée de Fourier

$$
\widehat{V}(\lambda)=\int_{-\infty}^{+\infty} \mathrm{e}^{-i t \lambda} V(t) \mathrm{d} t
$$

est bien définie pour $\operatorname{Im} \lambda<0$, comme opérateur borné de $H$. On a

$$
V(t)=(2 \pi)^{-1} \int_{\operatorname{Im} \lambda=-\varepsilon} \mathrm{e}^{-i t \lambda} \widehat{V}(\lambda) \mathrm{d} \lambda, \quad \forall \varepsilon>0
$$

et vérifie

$$
\left(\partial_{t}-i G\right) V(t)=\varphi^{\prime}(t) U(t) .
$$

Donc $\widehat{V}(\lambda)=i(G-\lambda)^{-1} \widehat{\varphi^{\prime} U}(\lambda)$ avec $\operatorname{Im} \lambda<0$.

Comme $\varphi^{\prime}(t) U(t) f$ est à support compact indépendant de $t$, alors $\widehat{\varphi^{\prime} U}(\lambda)$ : $H_{\text {comp }} \rightarrow H_{\text {comp }}$ est analytique sur $\mathbb{C}$. De plus $(G-\lambda)^{-1}: H_{\text {comp }} \rightarrow H_{\text {loc }}$ s'étend en un opérateur méromorphe sur $\mathbb{C}$ si $d$ est impair, et sur la surface de Riemann du logarithme, si $d$ est pair et on a

$$
(G-\lambda)^{-1}=-i\left(\begin{array}{cc}
R(\lambda)(2 a+i \lambda) & R(\lambda) \\
\lambda R(\lambda)(2 a+i \lambda)-\operatorname{Id} & i \lambda R(\lambda)
\end{array}\right) .
$$

Ainsi si $d$ est impair, (1.7) donne que

$$
\exists c>0 \quad \text { tel que } \quad\left\|(G-\lambda)^{-1} f\right\|_{R} \leq c\|f\|
$$

pour toute $f \in H$ telle que Supp $f \subset B_{R}$ et pour tout $\lambda \in \mathbb{C}$ tel que $\operatorname{Im} \lambda \leq \delta$.

Et d'après la remarque 2.1.1

$$
(G-\lambda)^{-1}=\mathcal{M}_{d}^{\prime} \lambda^{d-1} \log \lambda+O\left(|\lambda|^{d-1}\right), \quad \lambda \rightarrow 0,\left|\arg \lambda+\frac{1}{2} \pi\right| \leq \pi,
$$

modulo une fonction analytique en $\lambda=0$ si $d$ est pair.

TOME $131-2003-\mathrm{N}^{\mathrm{O}} 2$ 
D'après l'équation (3.1), on a

$$
\begin{gathered}
V(t) f=(2 \pi)^{-1} \int_{\operatorname{Im} \lambda=-\varepsilon} \mathrm{e}^{i t \lambda}(G-\lambda)^{-1} \widehat{\varphi^{\prime} U}(\lambda) f \mathrm{~d} \lambda, \quad \forall \varepsilon>0 \\
=(2 \pi)^{-1} \mathrm{e}^{-\delta t} \int_{-\infty}^{+\infty} \mathrm{e}^{i t z}(G-(z+i \delta))^{-1} \widehat{\varphi^{\prime} U}(z+i \delta) f \mathrm{~d} z \\
+(2 \pi)^{-1} \lim _{\varepsilon \rightarrow 0}\left\{\int_{\substack{\operatorname{Re} \lambda=-\epsilon, 0 \leq \operatorname{Im} \lambda \leq \delta}} \mathrm{e}^{i t \lambda}(G-\lambda)^{-1} \widehat{\varphi^{\prime} U}(\lambda) f d \lambda\right. \\
\left.\quad-\int_{\substack{\operatorname{Re} \lambda=\epsilon, 0 \leq \operatorname{Im} \lambda \leq \delta}} \mathrm{e}^{i t \lambda}(G-\lambda)^{-1} \widehat{\varphi^{\prime} U}(\lambda) f \mathrm{~d} \lambda\right\} \\
=(2 \pi)^{-1} \mathrm{e}^{-\delta t} W_{1}(t) f+W_{2}(t) f .
\end{gathered}
$$

- On examine d'abord $W_{2}(t) f$. Dans la première intégrale, $\lambda$ est paramétré par $\lambda=-\epsilon+i y$, et dans la deuxième intégrale, $\lambda=\epsilon+i y$. Il est clair que $W_{2}(t) f \equiv 0$ si $d$ est impair. Par contre pour $d$ pair, on a à partir de (3.4) et en remarquant que $|\log (-\epsilon+i y)-\log (\epsilon+i y)| \leq \mathrm{C}^{\text {te }}$, on voit aisément que $W_{2}(t)$ est en $O\left(t^{-d}\right)$. En d'autre termes,

$$
\left\|W_{2}(t) f\right\|_{R} \leq C t^{-d}\|f\| .
$$

- Pour estimer $\left\|W_{1}(t) f\right\|_{R}$, on va utiliser l'identité de Plancherel avec l'inégalité (3.3). On a

$$
\begin{aligned}
\int_{-\infty}^{+\infty}\left\|W_{1}(t) f\right\|_{R}^{2} \mathrm{~d} t & =\int_{-\infty}^{+\infty}\left\|(G-(z+i \delta))^{-1} \widehat{\varphi^{\prime} U}(z+i \delta) f\right\|^{2} \\
& \leq c \int_{-\infty}^{+\infty}\left\|\widehat{\varphi^{\prime} U}(z+i \delta) f\right\|_{R}^{2} \mathrm{~d} z \\
& =c \int_{-\infty}^{+\infty} \mathrm{e}^{2 \delta t}\left\|\varphi^{\prime}(t) U(t) f\right\|_{R}^{2} \mathrm{~d} t \mathrm{~d} z \\
& \leq c\|f\|^{2}
\end{aligned}
$$

Soit $\chi \in C_{0}^{\infty}\left(\mathbb{R}^{d}\right)$ tel que $\chi=1$ sur $|x| \leq R$. On a

$$
\begin{aligned}
\left(\partial_{t}-i G\right) \chi W_{1}(t) f & =\delta \chi W_{1}(t) f-i \chi \int_{-\infty}^{+\infty} \mathrm{e}^{-i t z \widehat{\varphi^{\prime} U}}(z+i \delta) f \mathrm{~d} z \\
& =\tilde{W}_{1}(t) f
\end{aligned}
$$

$$
\left\|W_{1}\left(t_{0}\right) f\right\|_{R} \leq c\|f\|
$$

On a

$$
\chi W_{1}(t) f=U(t) \chi W_{1}\left(t_{0}\right) f+\int_{t_{0}}^{t} U\left(t-t_{0}-s\right) \tilde{W}_{1}(s) f \mathrm{~d} s
$$

BULLETIN DE LA SOCIÉTÉ MATHÉMATIQUE DE FRANCE 


$$
\begin{aligned}
\left\|W_{1}(t) f\right\|_{R} & \leq\left\|\chi W_{1}(t) f\right\| \leq c\|f\|+\int_{t_{0}}^{t}\left\|\tilde{W}_{1}(s) f\right\| \mathrm{d} s \\
& \leq c\|f\|+c t^{\frac{1}{2}}\left(\int_{-\infty}^{+\infty}\left\|\tilde{W}_{1}(s) f\right\|^{2} \mathrm{~d} s\right)^{\frac{1}{2}} \\
& \leq c t^{\frac{1}{2}}\|f\|, \quad t \geq 1 .
\end{aligned}
$$

Ainsi (1.8) et (1.9) se déduisent de (3.6), (3.7) et (3.15).

\section{Le meilleur taux de décroissance en dimension impaire}

Soit $\beta$ le meilleur taux de décroissance exponentielle défini par

$$
\begin{aligned}
\beta=\sup \{\delta>0 ; & \exists c>0, \forall f \in H, \operatorname{Supp} f \subset B_{R}, \\
& \left.E_{R}(U(t) f) \leq c \mathrm{e}^{-\delta t} E(U(0) f), \forall t \geq 0\right\} .
\end{aligned}
$$

Preuve du théorème 1.3. - D'après le théorème 1.0.2, on a

$$
\beta \geq \alpha=2 \min (D(0), C(\infty)) .
$$

Montrons que $\beta \leq \alpha$.

- On a $\beta \leq 2 D(0)$. - En effet, supposons que $2 D(0)<\beta$; alors il existe $\lambda_{1}$ pôle de $R(\lambda)$ tel que $2 \operatorname{Im} \lambda_{1}<\beta$. On a $R\left(\lambda_{1}\right) f=\int_{0}^{+\infty} \mathrm{e}^{-i \lambda_{1} t} u(t) f \mathrm{~d} t$, donc

$$
\begin{aligned}
\left\|R\left(\lambda_{1}\right) f\right\|_{R} & \leq \int_{0}^{+\infty} \mathrm{e}^{\operatorname{Im} \lambda_{1} t}\|u(t) f\|_{R} \mathrm{~d} t \\
& \leq \int_{0}^{+\infty} \mathrm{e}^{\operatorname{Im} \lambda_{1} t} \mathrm{e}^{-\frac{1}{2} \beta t}\|f\| \mathrm{d} t \\
& \leq \int_{0}^{+\infty} \mathrm{e}^{\frac{1}{2} t\left(2 \operatorname{Im} \lambda_{1}-\beta\right)}\|f\| \mathrm{d} t \\
& \leq-\frac{\|f\|}{\operatorname{Im} \lambda_{1}-\frac{1}{2} \beta}<\infty, \quad \forall f \in H_{R} .
\end{aligned}
$$

Donc $\lambda_{1}$ n'est pas un pôle de $R\left(\lambda_{1}\right)$, ce qui est absurde.

- On a $\beta \leq 2 C(\infty)$. - En supposant que $2 C(\infty)<\beta$, il existe $\eta>0$ tel que $\beta=2 C(\infty)+4 \eta$ et il existe $c>0$ tel que pour tout $f \in H_{R}$ et tout $t>0$,

$$
E_{R}(u(t)) \leq c \mathrm{e}^{-(\beta-\eta) t} E(0) .
$$

Soit $t>0$ tel que $c \mathrm{e}^{-(\beta-\eta) t_{0}} \leq \mathrm{e}^{-(\beta-2 \eta) t_{0}}$

$$
\begin{aligned}
E_{R}\left(u\left(t_{0}\right)\right) & \leq \mathrm{e}^{-(\beta-2 \eta) t_{0}} E(0) \quad \forall f \in H_{R}, \\
& \leq \mathrm{e}^{-2(C(\infty)+\eta) t_{0}} E(0) .
\end{aligned}
$$

TOME $131-2003-\mathrm{N}^{\mathrm{O}} 2$ 
Comme $C(\infty)<\infty$ (sinon l'inégalité à démontrer est vraie) et $C\left(t_{0}\right) \leq C(\infty)$, il existe $\rho_{0}$ tel que

$$
\begin{gathered}
C\left(t_{0}\right)=\frac{1}{t_{0}} \int_{0}^{t_{0}} a\left(x\left(s, \rho_{0}\right)\right) \mathrm{d} s \leq \frac{\beta}{2}-\eta \quad\left(\rho_{0} \text { captif }\right) \\
E_{R}\left(u\left(t_{0}\right)\right) \leq \mathrm{e}^{-2\left(C\left(t_{0}\right)+\eta\right) t_{0}} E(0)
\end{gathered}
$$

ce qui est absurde. En effet, soit $f_{k} \underset{L^{2}}{\rightarrow} 0$ tel que $\left\|f_{k}\right\|_{L^{2}}=1$. On note $u_{k}$ la solution de (1.3) avec $u_{k}(0)=0, \partial_{t} u_{k}(0)=f_{k}$ et soit $\mu$ une mesure de défaut microlocale assoociée à $\left(u_{k}\right)$ dans $H$. La fonction $f_{k}$ est choisie telle que $\mu$ soit portée par $\gamma$ (rayon bicaractéristique issu de $\rho_{0} \subset B_{R}$ ).

On note $u_{s}(t, x)=u(t+s, x), s \in[0, \varrho], \varrho>0$,

$$
\begin{aligned}
& \int_{0}^{\varrho} E_{R}\left(u_{s}\left(t_{0}\right)\right) \mathrm{d} s \leq \mathrm{e}^{-2\left(C\left(t_{0}\right)+\eta\right) t_{0}} \int_{0}^{\varrho} E\left(u_{s}(0)\right) \mathrm{d} s \\
& \mu(] t_{0}, t_{0}+\varrho[) \leq \mathrm{e}^{-2\left(C\left(t_{0}\right)+\eta\right) t_{0}} \mu(] 0, \varrho[) \\
& \mathrm{e}^{-\frac{2}{t_{0}} \int_{0}^{t_{0}} a\left(x\left(s, \rho_{0}\right)\right) \mathrm{d} s} \mu(] 0, \varrho[) \leq \mathrm{e}^{-\frac{2}{t_{0}} \int_{0}^{t_{0}} a\left(x\left(s, \rho_{0}\right)\right) \mathrm{d} s-2 \eta t_{0}} \mu(] 0, \varrho[) .
\end{aligned}
$$

Or $\mu(] 0, \varrho[) \neq 0$ (sinon $u_{k}$ tend vers zéro dans $H^{1}(] 0, \varrho\left[\times B_{R}\right)$, ce qui contredit $\left.\left\|f_{k}\right\|_{L^{2}}=1\right)$. Cela achève la preuve du théorème 1.0.3.

Remerciements. - Je remercie le Professeur Nicolas Burq, pour les discussions que j'ai eues avec lui à propos de cet article lors de mes séjours à Orsay. Je remercie également le Professeur Gilles Lebeau pour ses remarques fructueuses qui ont permis d'améliorer le contenu de ce travail.

\section{BIBLIOGRAPHIE}

[1] Aloui (L.) \& Khenissi (M.) - Stabilisation de l'équation des ondes dans un domaine extérieur, Rev. Mat. Iberoamericana, t. 28 (2002), pp. 1-16.

[2] Burq (N.) - Décroissance de l'énergie locale de l'équation des ondes pour le problème extérieur, Acta Math., t. 180 (1998), pp. 1-29.

[3] GÉRARD (C.) - Asymtotique des pôles de la matrice de scattering pour deux obstacles strictement convexe, Mém. Soc. math. France (N.S.), vol. 31, Société Mathématique de France, Paris, 1988.

[4] GÉrard (P.) - Microlocal defect measures, Comm. Partial Differential Equations, t. 16 (1991), pp. 1761-1794.

[5] Gohberg (I.C.) \& Krein (M.G.) - Introduction to the Theory of Linear non Self adjoint Operators, Translations of Mathematical Monograph, vol. 18, Amer. Math. Soc., 1969.

[6] Lax (P.D.) \& Phillips (R.S.) - Scattering theory, Pure and Applied Mathematics, vol. 26, Academic Press, New York, 1967.

BULletin DE LA SOCIÉtÉ MATHÉMATIQUE DE FRANCE 
[7] The acoustic equation with an indefinete energy form end the Schrödinger equation, J. Funct. Anal., t. 1 (1967), pp. 37-83.

[8] _ Scattering theory for the Acoustic Equation in an Even Number of Space Dimensions, Indiana Univ. Math. J., t. 22 (1972), no. 2, pp. 101-133.

[9] Lebeau (G.) - Équation des ondes amorties, in Algebraic and Geometric Methods in Mathematical Physics, Kluwer Academic Publishers, Nertherlands, 1996, pp. 73-109.

[10] Melrose (R.) - Singularities and energie decay in acoustical scattering, Duke Math. J., t. 46 (1979), pp. 43-59.

[11] Morawetz (C.S.) - Decay for solutions of the exterior problem for the wave equation, Comm. Pure Appl. Math., t. 28 (1975), pp. 229-264.

[12] Ralston (J.) - Solution of the wave equation with localized energy, Comm. Pure Appl. Math., t. 22 (1969), pp. 807-823.

[13] TARTAR (L.) - H-mesures : a new approach for studying homogenization, oscillations and concentration effects in partial differential equations, Proc. Roy. Soc. Edinburgh Sect. A, t. 115 (1993), pp. 193-230.

[14] VAinberg (B.) - Asymtotic methods in equations of mathematical physics, Gordon and Breach, New York, 1988.

[15] Vodev (G.) - Sharp Bounds on the Number of Scattering Poles for Perturbations of Laplacian, Comm. Math. Phys., t. 146 (1992), pp. 205-216.

[16] _ On the uniform decay of the local energy, Serdica Math. J., t. 25 (1999), no. 3, pp. 191-206. 